ORIGINAL ARTICLE

\title{
CT or not CT - that is the question. Whether 'tis better to evaluate clinically and $x$ ray than to undertake a CT head scan!
}

\author{
D M Macgregor, L McKie
}

Emerg Med J 2005;22:541-543. doi: 10.1136/emj.2004.017160

See end of article for authors' affiliations

a.r.

Correspondence to: Dr Diana M Macgregor, Accident \& Emergency

Department, Royal

Aberdeen Children's

Hospital, Foresterhill,

Aberdeen, AB25 2ZG,

Scotland;

diana.macgregor@

nhs.net

Accepted for publication 12 July 2004
Objective: To evaluate the usage of computed tomography (CT) head scanning in children at the Royal Aberdeen Children's Hospital after the publication of the National Institute of Clinical Excellence (NICE) guidelines on the management of head injury.

Methods: The Accident and Emergency case records of all children presenting with a head injury over a three month period were reviewed and the number of attendances, radiographs, and CT head scans undertaken were noted. Also noted was the number of additional CT head scans that would have been performed if the NICE guidelines had been rigidly followed.

Results: Five hundred and thirty seven children were included in the study: $67 \%$ were boys. Two hundred and ten (39\%) had skull radiographs: six demonstrated skull fractures and eight (1.5\%) underwent CT head scan, with one positive report of a skull fracture. There were no reports of intracranial abnormalities. Ninety nine (18.4\%) were admitted. Strictly applying all the NICE criteria for CT scanning would have resulted in an additional 54 patients being scanned.

Conclusion: Rigid adherence to the NICE guidelines in all children with head injuries would have resulted in an almost eightfold increase in CT head scans performed. None of these children had clinical signs of intracranial injury and would have been exposed to a large amount of ionising radiation. The use of guidelines in practice must always be considered in conjunction with clinical judgement.
$\mathrm{H}$ ead injury is one of the most common reasons for children to present to medical services for urgent care in the UK. Childhood head injury is a major public health problem, accounting for $15 \%$ of deaths in the $1-15$ year age group and $25 \%$ of deaths in the $5-15$ year age group. ${ }^{1}$ Early diagnosis and treatment of patients with head injury improves the morbidity and mortality associated with any intracranial injury. ${ }^{2}$ Whether to perform skull radiographs and/or computed tomography (CT) of the head is an area of controversy and debate, with a vast amount of literature published on this subject.

Differing guidelines on the management of head injuries have been developed but none are specifically for paediatric practice. Published in June 2003, the National Institute for Clinical Excellence (NICE) guideline sets out Recommendations for NHS care of people who have suffered a head injury. ${ }^{3}$ This guideline provides some differentiation between the risk factors in adults and children (Appendix 1) and suggests a system for selection of patients for CT head imaging. Unfortunately even this system is open to subjectivity, especially in children, in particular the "vomiting" criterion:

\footnotetext{
"More than one episode of vomiting (clinical judgement should be used regarding the cause of vomiting in those aged 12 years or younger, and whether imaging is necessary) $)^{\prime \prime}$.

Another criterion that is subjective in children is:

"Dangerous mechanism of injury la pedestrian struck by a motor vehicle, an occupant ejected from a motor vehicle, or a fall from a height of greater than $1 \mathrm{~m}$ or five stairs). A lower threshold for height of falls should be used when dealing with infants and young children-that is, aged under 5 years".
}

It can be difficult to judge what is "dangerous" and such factors should always be considered in conjunction with clinical findings, although a fall of over $5 \mathrm{~m}$ has been shown to pose the greatest significance to morbidity and mortality in children. ${ }^{4}$

Eighty per cent of presentations of head injuries in all age groups (children and adults) are due to minor head injury, ${ }^{5}$ but an injury cannot be classed as minor until all relevant risk factors have been excluded and it is more important to identify patients who are at risk of intracranial injury than to seek to place head injuries into categories. Evaluation of a young child can be difficult, especially when an incident is not witnessed or the history unclear. Thorough examination is essential in conjunction with the utilisation of the paediatric version of the Glasgow Coma Scale (GCS) where appropriate. If there is clinical suspicion of skull fracture then skull radiographs should be considered because, in children, significant intracranial injury occurs more frequently in association with a skull fracture than in adults. ${ }^{6}$ The Royal College of Surgeons Working Party Report on the management of head injuries recommends the following indications for skull $x$ ray:

\begin{abstract}
"History of loss of consciousness or amnesia; scalp laceration (to bone or greater than $5 \mathrm{~cm}$ long), bruise or swelling; violent mechanism of injury; persistent headache; or significant maxillofacial injuries. In addition to this, for an infant: fall from a significant height la fall greater than twice the height of the child may be significant); a fall on to a hard surface; tense fontanelle; or suspected non-accidental injury".?
\end{abstract}

Abbreviations: A\&E, Accident and Emergency; $C T$, computed tomography; GCS, Glasgow Coma Scale; NICE, National Institute of Clinical Excellence; RACH, Royal Aberdeen Children's Hospital 
The presence of a skull fracture is important if CT scanning is not being considered initially, as the incidence of intracranial pathology in children with a skull fracture has been reported as 1 in 157. ${ }^{1}$ Patients with clinical signs of a fracture may not undergo a CT head scan as a first line investigation but this should be considered if there is suspicion of intracranial injury. CT scanning is a reliable, non-invasive investigation, ${ }^{8}$ which is available on an urgent basis 24 hours a day in the Royal Aberdeen Children's Hospital (RACH).

The aim of this study was to evaluate current CT imaging rates and adherence to the NICE guidelines on CT scanning in our department.

\section{METHODS}

The Accident and Emergency (A\&E) department at RACH is the only paediatric A\&E department in the north east of Scotland, serving a population of over half a million. Annually the department sees over 20000 new cases, all under the age of 14 years.

Every child presenting with a head injury between the 1 July and the 30 September 2003 had their case record reviewed retrospectively. Children with facial injuries were excluded. Age, sex, history, and significant clinical findings were noted. All children with head injuries under 14 years of age were included and each history noted mechanism of injury (fig 1) and pertinent symptoms. Symptoms such as loss of consciousness, amnesia, vomiting, seizure, drowsiness, and confusion were recorded. Significant clinical findings included GCS, scalp haematoma, otorrhoea, epistaxis, and focal neurological signs. All radiographs and CT head scans performed were also noted. Children referred for CT head scan were also cross referenced with the CT department's log. In addition, every patient fulfilling the specific CT scanning criteria as defined by the NICE guidelines was also noted in order to asses how many additional CT head scans that would have been performed if the NICE guidelines had been followed rigidly.

\section{RESULTS}

In the three month audit period, 4639 new cases attended A\&E and, of these, $569(12.2 \%)$ presented with a head injury. Thirty two patients whose case records could not be located were excluded, resulting in 537 cases being included in the study. Three hundred and sixty (67\%) were male and 99 (18.4\%) were admitted. Two hundred and ten (39\%) underwent skull radiographs, with six of these demonstrating a skull fracture. Eight (1.5\%) underwent a CT head scan. Four of these children also had skull radiographs, two of which were reported as showing skull fractures-both gave a history of GCS $<13$ following the head injury. Of the eight CT head scans, the only positive finding was one parietal skull fracture. No intracranial abnormalities were reported.

If there had been stricter adherence to the NICE clinical practice guidelines, excluding the "vomiting criterion", an additional 15 CT head scans would have been undertaken. This increases the number of patients undergoing the investigation from eight $(1.5 \%)$ to $23(4.3 \%)$, which would have resulted in a 2.5 times increase in the CT head scanning rate. If the "vomiting" criterion had also been strictly applied in our study, then a further 39 CT scans would have been required. Together with the previously identified 23 children, this would give a total of 62 patients who would have been imaged —an almost eightfold increase.

\section{DISCUSSION}

Being a major cause of death and disability, head injury in children is recognised as a significant public health problem, which poses substantial demands on a wide variety of health services. ${ }^{9}$ A recent study carried out in RACH, looking at A\&E attendances in children under one year of age, found that of 434 patients attending as a result of an "accident" over half were head injuries. ${ }^{10}$

In our study, eight (1.5\%) of the 537 patients attending with head injury underwent CT head scan, with none demonstrating an intracranial abnormality. This is a lower scanning rate than in other studies and Lloyd et al reported a higher rate (15\%) of abnormalities. ${ }^{11}$ Of the 15 children who were recorded as having clinical findings that fitted the NICE guidelines, excluding the "vomiting' criterion", but were not scanned, 10 (66\%) underwent skull radiography. All these were reported as normal. Forty per cent of these children were not admitted. This implies that a large proportion was either considered well enough to be sent home or that clinically no imaging was thought necessary. None of these patients returned and it is presumed that there were no sequelae. If these patients had undergone a CT head scan the rate of scanning would increase from $1.5 \%$ to $4.3 \%$.

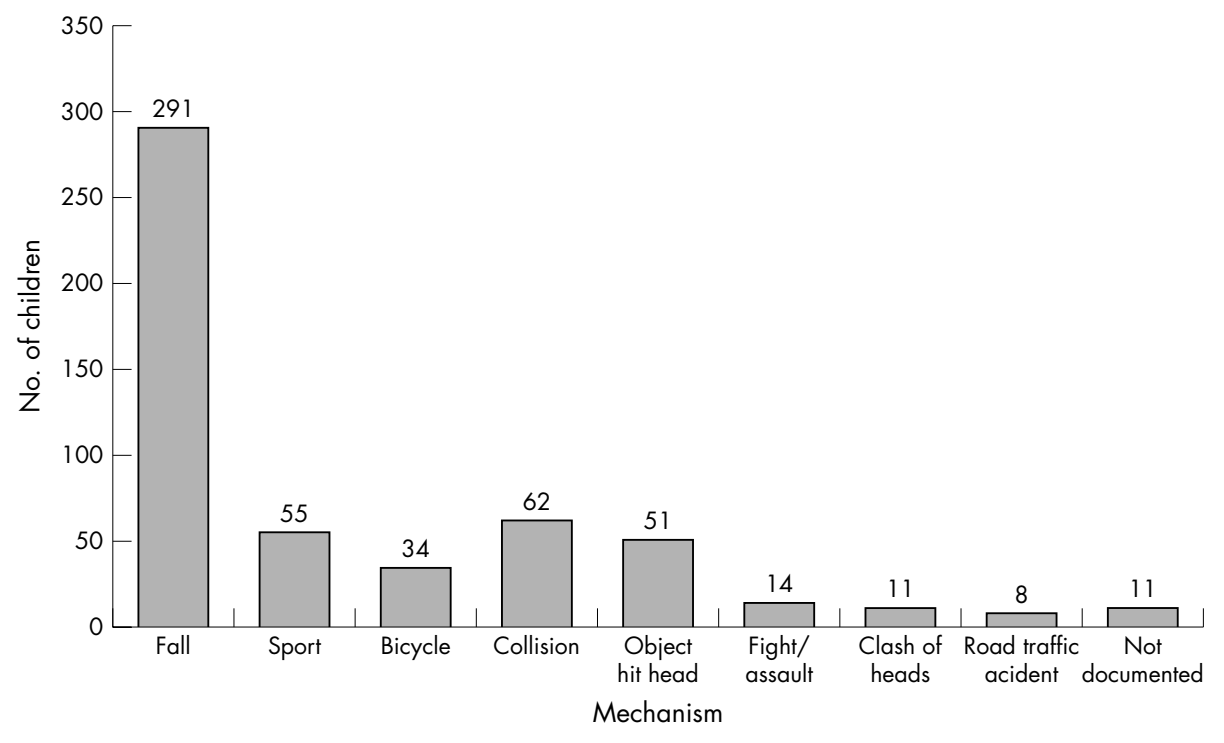

Figure 1 Mechanisms of injury 
When considering the guideline criterion on vomiting in children it can be seen that, if followed rigidly, a further 39 scans would have been indicated, which increases the CT head scan rate to $11.5 \%$ of the cohort. This rate is high considering the low yield of positive findings. It would seem that, quite correctly, the guidelines suggest that vomiting in children with head injury must be judged along with the complete clinical picture. It is known that vomiting in children may not be as reliable a sign of severe head injury as in adults, ${ }^{5}$ and this is especially true in infants when a history of greater than one vomit after head injury within one hour of eating is usually benign. ${ }^{1}$

Of the eight patients that were actually CT scanned, four also underwent a skull radiograph with two of these demonstrating a skull fracture. Only one of these fractures was noted on the CT scan. Bony injuries are not always demonstrated on CT scan and hence it is possible that the four patients who underwent CT scanning but not skull radiography may have had undiagnosed fractures. In practice, if a patient has signs of any intracranial injury, then a CT head scan would be the main investigation of choice.

Sixty per cent of the unscanned but "NICE positive" group were admitted to hospital. However, if they had all undergone CT scanning we can presume that they would all have been admitted. This has obvious implications for bed space, staffing resource, and also child and parental anxiety.

CT is the gold standard investigation for identifying intracranial injury following head trauma but is not without limitations. Staff must be aware of the radiation risks that CT scanning poses-one CT head scan exposes a child to approximately $0.5 \mathrm{mGy}$, which is the equivalent of about 100 chest $x$ rays. ${ }^{12}$ In the USA, $33 \%$ of all paediatric CT scans are performed within the first 10 years of life, when children are most susceptible to radiation damage. ${ }^{13}$ The importance of limiting unnecessary radiation in children was highlighted in a recent Swedish study, which demonstrated that there may be long term cognitive effects for children exposed to ionising radiation. ${ }^{14}$ There are also financial implications: one American study suggested that the cost of a CT scan would be around $\$ 500-1000(£ 350-650) .^{12}$

Disadvantages of this study are the very small numbers of patients who actually underwent CT head scan at RACH and the limited study period.

\section{CONCLUSION}

This study demonstrates that, if the NICE guidelines were rigidly applied, the rates of CT head scans in children would increase considerably. It should be remembered that although guidelines are produced to aid staff they should only act as guidance and clinical judgment should always be used in conjunction with such recommendations. However any departure from recommendations made in evidence based guidelines must be considered carefully particularly when there may be medico-legal implications.

\section{ACKNOWLEDGEMENTS}

I would like to acknowledge all the time and the hard work put in by Laurin McKie who researched this information as part of her elective project.

\footnotetext{
Authors' affiliations

D M Macgregor, Accident \& Emergency Department, Royal Aberdeen Children's Hospital, Aberdeen, Scotland

L McKie, Aberdeen University School of Medicine, Aberdeen, Scotland

Competing interests: none declared.
}

\section{REFERENCES}

1 Glasgow JF, McGovern SJ. Imaging the less seriously head injured child. Arch Dis Child 2000:82:333.

2 Quayle KS, Jaffe DM, Kuppermann N, et al. Diagnostic testing for acute head injury in children: when are head computed tomography and skull radiographs indicated? Pediatrics 1997;99(5):E1 1.

3 National Institute for Clinical Excellence. Clinical Guideline 4. Head injury triage-assessment, investigation and early management of head injury in infants, children and adults. June 2003

4 Beattie TF. Minor head injury. Arch Dis Child 1997;77:82-5.

5 Brookes M, MacMillan R, Cully S, et al. Head injuries in accident and emergency departments. How different are children from adults? J Epid Comm Health 1990;44:147-51.

6 Royal College of Paediatrics and Child Health. Guideline for good practice. Early management of patients with head injury. September 2001.

7 Royal College of Surgeons of England. Report of a working party on the management of head injuries. London: Royal College of Surgeons of England, 1999.

8 Kelly A, Kerr D. Are too many head CT scans ordered in emergency departments? Emer Med 2000;12:50-4.

9 Jennett B. Epidemiology of head injury. Arch Dis Child 1998;78:403-6.

10 Macgregor $\mathbf{D}$. Accident and emergency attendances by children under the age of 1 year as a result of injury. Emerg Med J 2003;20:21-4.

11 Lloyd D, Carty H, Patterson M, et al. Predictive value of skull radiography for intracranial injury in children with blunt head injury. Lancet 1997;349:821-4.

12 Herbert $M$. Issues in minor head injury: to $C T$ or not to $C T$, that is the question. Emerg Radiol 2000;7:7-13.

13 Frush DP, Donnelly LF, Rosen NS. Computed tomography and radiation risks: what pediatric health care providers should know. Pediatrics 2003;112:951-7.

14 Hall P, Adami HO, Trichopoulos P, et al. Effect of low doses of ionising radiation in infancy on cognitive function in adulthood: Swedish population based cohort study. Br Med J 2004;328:19-21.

\section{APPENDIX 1}

\section{SELECTION OF PATIENTS FOR CT IMAGING OF THE HEAD}

1.4.2.7 Patients who have sustained a head injury and present with any one of the following risk factors should have CT scanning of the head immediately requested.

- GCS less than 13 at any point since the injury.

- GCS equal to 13 or 14 at two hours after the injury.

- Suspected open or depressed skull fracture.

- Any sign of basal skull fracture (haemotympanum, "panda" eyes, cerebrospinal fluid otorrhoea, Battle's sign).

- Post-traumatic seizure.

- Focal neurological deficit.

- More than one episode of vomiting (clinical judgement should be used regarding the cause of vomiting in those aged 12 years or younger, and whether imaging is necessary).

- Amnesia for more than 30 minutes of events before impact. The assessment of amnesia will not be possible in pre-verbal children and is unlikely to be possible in any child aged under 5 years.

1.4.2.8 CT should also be immediately requested in patients with any of the following risk factors, provided they have experienced some loss of consciousness or amnesia since the injury:

- Age $\geqslant 65$ years.

- Coagulopathy (history of bleeding, clotting disorder, current treatment with warfarin).

- Dangerous mechanism of injury (a pedestrian struck by a motor vehicle, an occupant ejected from a motor vehicle, or a fall from a height of more than $1 \mathrm{~m}$ or five stairs). A lower threshold for height of falls should be used when dealing with infants and young children-that is, aged under 5 years. 